\title{
PRESENT SWISS CONCEPTS OF RHEUMATISM AND PHYSICAL MEDICINE*
}

\author{
BY \\ VICTOR OTT \\ From the University Institute for Physical Therapy, Zurich $\dagger$
}

This paper is intended as a report on some results of recent work in physical medicine and rheumatology in Switzerland. Since 1940 education in physical medicine, including medical hydrology and climatology, has been compulsory for all medical students. Thus there are chairs for physical medicine in all medical faculties; three of these are professorships, yet several institutes for physical therapy are dependent upon other hospital departments. At Geneva and Basle there are separate departments; but only at Zurich is there a fully independent clinical institute with departments for in-patients and out-patients.

As the recent investigations of the Institute for Physical Therapy at Zurich represent an important part of the work done in the field of physical medicine and rheumatology in Switzerland during the past years, I shall give a brief survey of this work. The chronic rheumatic diseases are a very important object of physical therapy; later on we shall speak about the reasons for this fact. Thus, an essential part of the work on which I have to report deals with problems of rheumatism.

\section{The Importance of Rheumatism in Public Health and Social Medicine in Switzerland}

The importance of rheumatic diseases in the public health and national economy of Switzerland was shown by the investigations of Bruck (1939). Some of the essential results of this extensive work are given in the following summary.

In the group of the employees of the Swiss Federal Railways, i.e. more than 10,000 men between 15 and 64 years of age, there were in one year 1.14 days of illness per 100 working days on account of rheumatism, but only 0.14 days of illness on account of tuberculosis. The full invalidism rate is 3.6 days for rheumatism, but only $\mathbf{0 . 0 7}$ days for tuberculosis. Thus, the ratio of invalidism on account of rheumatism compared with that of tuberculosis was as high as $51: 1$; in those cases who went back to work, the length of treatment, compared with tuberculosis, gave a ratio of 7.9 to 1 . With suitable methods it was possible to make from these statistics an approximate survey of the economic consequences of

\footnotetext{
- Lecture delivered at the Annual General Meeting of the International Society of Medical Hydrology, Buxton, October 6, 1946.

$\uparrow$ Prof. K. von Neergaard, Director.
}

rheumatism for the whole working population of Switzerland between the ages of 15 and 64 years. In 1934-5 the treatment costs for rheumatic diseases were 18 million Swiss francs, the loss from transitory incapacity 66 millions, and the loss from early invalidism about 155 million. Considering the devaluation of money, the result at the present time would be an annual loss of at least $\mathbf{3 3 0}$ million Swiss francs, i.e. about $£ 19,000,000$ caused by rheumatic diseases.

The survey of the years 1925 to 1935 showed a somewhat different, but equally tremendous average result: morbidity and incapacity rates on account of rheumatism were as high as $21 \%$, the tuberculosis morbidity as low as $0.59 \%$. Thus, in these years, the morbidity from rheumatism was 36 times greater than that from tuberculosis, while the treatment time was 4.5 times greater.

These figures take into consideration only peripheral rheumatism. But diseases of the circulatory system play a great part in morbidity and mortality; the latter is in this country about $50 \%$ higher than for tuberculosis and cancer together. It is certain that a high percentage of these diseases are caused by rheumatism (Dietrich, 1938; Edström, 1935), but until now there has been no safe basis for an exact statistical investigation of this problem.

Furthermore, Bruck's investigations showed the importance of harmful conditions in various types of work for the pathogenesis of rheumatic diseases, whereby the injury seems mostly to be caused by cold, wet, and draughts.

Finally, the allocation of health insurance money for the treatment of rheumatic diseases is much smaller than that for tuberculosis. In spite of the fact that the morbidity is much greater, the patients suffering from rheumatism have not yet got the same financial help for treatment as those suffering from tuberculosis.

Thus, these investigations demonstrate the enormous importance of rheumatism to the public health in Switzerland. Beyond their purely scientific interest, they also have practical consequences: firstly, they have stimulated the interest of a wide group of the medical profession, and they have been responsible for an increase in the efforts to improve the prophylaxis and treatment of rheumatism within the framework of social medicine. 
Furthermore, they have demonstrated to the public the important rôle of physical medicine, and, finally, they have shown the great necessity for medical research in this field of pathology.

\section{Clinical Investigations on Rheumatism Problems}

From the clinical point of view, the problem has been especially treated by von Neergaard (1939). Besides papers on detailed clinical subjects of rheumatism, this author has dealt particularly with the question of the aetiology of rheumatism, a problem that is as important for prophylaxis as for treatment in rheumatic diseases. This work was essentially based upon clinical facts that may be found in the individual patient. The principal results which concern at any rate those patients living in our region and coming for treatment to our clinical departments may be summarized as follows.

Remissions and exacerbations.-From the clinical point of view, the term "rheumatism" as a symptomatological term for all those diseases characterized by wandering pains in the joints and fibrous tissues is insufficient. It is preferable to call conditions of specific aetiology causing similar symptoms by the name of the disease concerned. They are a part of the differential diagnosis, but they are not identical with the rheumatic diseases themselves. The diagnosis of rheumatism is based upon the characteristic signs of the rheumatic disease itself. These characteristics are to be found not only in the actually existing state of the organs, but also in the peculi- arities of the pathological course. These peculiarities are, above all, the change between remissions and exacerbations, as well as the great dependence of the conditions upon physical influences, such as weather, environment, and employment traumata. The diagnostic considerations comprising the factor of time become, therefore, dynamic, and are superior to the mere static concept.

Focal infection.-Focal infection with streptococci does exist, but it is a relatively rare condition. In most cases it may be differentiated from essentially rheumatic diseases by the peculiarities of its clinical picture. The following facts support this statement. Surgical treatment of the so-called foci is a failure in the majority of cases. Furthermore, chronic infections with streptococci take a much steadier course, they are less dependent on exterior physical agents, and they can be healed by removing the focus. Finally, their haematological and radiological changes, are different from those in typical rheumatic cases.

Allergy.-The term " allergy" should not be used as a slogan to explain rheumatic conditions. There are several weighty arguments against the theory that rheumatism is nothing but an allergic tissue reaction to absolutely non-specific allergenic factors; a theory which was particularly represented by Klinge (1933). First we must remember that Aschoff (1934), who found rheumatic granuloma, held the opinion that the granulomas produced by non-specific sensitization are not identical with those found in rheumatic fever. On the other hand it is very difficult to explain rheumatism as a merely allergic tissue reaction, since the clinical picture is so well-defined and characteristic, and since other pathological conditions with a specific clinical picture are generally produced byspecific aetiology.

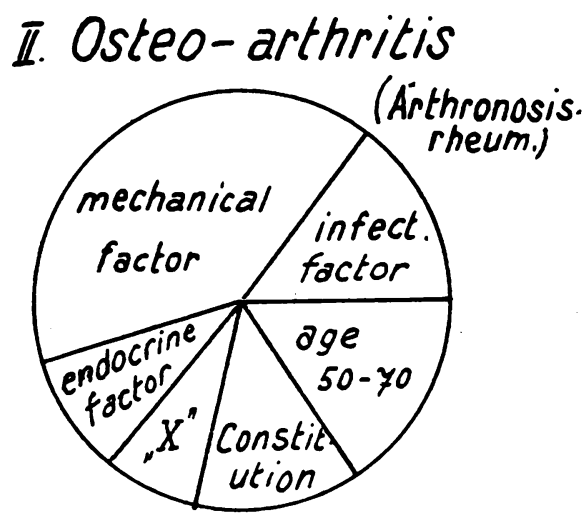

Asthenic type.

Pyknic type.

\section{Rheumatoid arthritis}

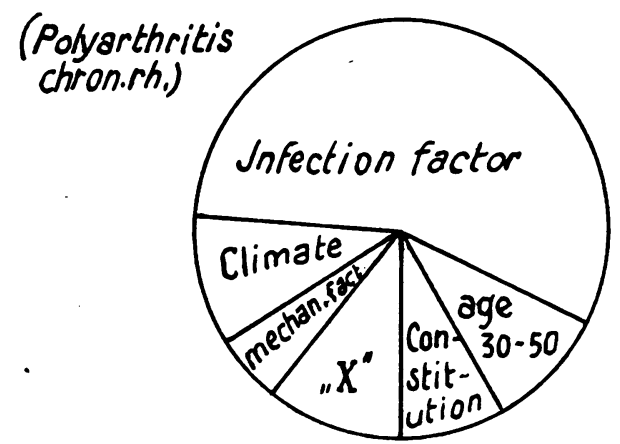

Fig. 1.-Spectra of pathogenic factors in various forms of chronic rheumatism (after von Neergaard, 1939). 
Virus infection.-Naturally, allergy, i.e. a change of pathological reaction, plays a certain part in a chronic disease like rheumatism, as well as in tuberculosis, etc. Nevertheless the clinical worker may put the question, which pathagenic factor is most important for the sensitization? The history of rheumatic patients now shows a remarkable coincidence in the time of onset of rheumatic exacerbations with catarrh of the respiratory tract and with influenza. There seem to be, clinically as well as serologically, connexions between the common cold and influenza both of which are virus diseases. Von Neergaard, therefore, holds them to be a common group of diseases. On the basis of a thorough clinical study, the author regards "vulgar" rheumatism, in its peripheric as well as in its visceral form, as a chronic, systemic form of this cold-influenza-infection.

It must, however, be taken into consideration that the aetiology of rheumatism is not a simple, directly caused one. We find, on the contrary, a spectrum of factors, in which the importance of each factor is variable (Fig. 1). Sometimes the infection is most important; in other cases it is the constitution, endocrine disorders, or physical environmental agents. The factor of infection seems always to be indispensable, but the outbreak of the disease itself takes place only when various conditions coincide. The pathogenesis of rheumatism is, therefore, absolutely conditional.

Infection with the common cold virus is quite ubiquitous in our latitudes. Large-scale prophylaxis is, therefore, out of the question. Furthermore, immunity against this illness is only relative: there is no permanent immunity. Remissions and exacerbations in the chronic course of the disease depend largely on physical (mostly thermic) factors. For this reason we speak of this special group of diseases as " colds".

\section{The Importance of Physical Medicine in the Treatment of Rheumatism}

The prophylaxis and treatment of colds and rheumatism does not, however, consist only of avoiding physical noxiousness: experience shows that by physical treatment, especially with thermotherapy and hydrotherapy, these diseases can be influenced more effectively in the majority of cases than by medical applications; it may even be said that physical medicine is here the supreme method of treatment; but we need to dispute this claim more precisely. In spite of numerous investigations into certain physiological reactions on physical treatment, we have hitherto been at a loss to explain theoretically the empirical efficiency of physical therapy in the case of certain infectious diseases. The following researches are a small contribution towards the solution of this problem.

For the last few years, the writer has been trying to explain by experimental methods the efficacy of the Finnish Sauna bath. Its effects may be regarded as characteristic of physical methods. In this respect we should, however, not lay too much stress on the various single results, but essentially on their basic evaluation. The Finnish Sauna bathris a hot-air bath with a temperature from 60 to $90^{\circ} \mathrm{C}$. $\left(194^{\circ} \mathrm{F}\right.$.). The dampness of the air is, on the whole, very low ( 5 to $15 \%$, estimated with the wet bulb), but by means of steam production it can be increased considerably for short intervals. After a bathing time of about 10 to 30 minutes, a short refrigerant with cold water is taken, in winter even with snow.

In the heated room of the Sauna not only is an intensive sweating taking place, but also a slight hyperthermia. The body-temperature may rise within 20 minutes to

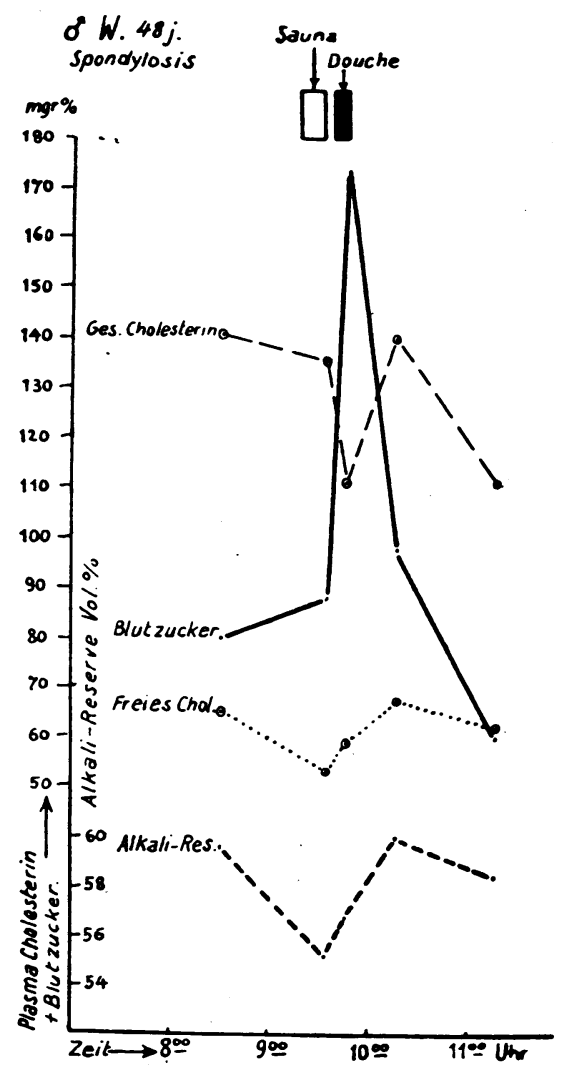

FIG. 2.-Influences of a Sauna bath followed by cold douche, on blood-sugar, plasma cholesterol, and alkaline reserve. Male patient, aged 48 years, suffering from osteo-arthritis of the spine, fasting. The blood-sugar curve shows a short but intense sympatheticotonic stimulus, with its maximum immediately after the cold douche. Note the biphasic form of cholesterol and alkaline reserve curves, showing the same effect. The fall of alkaline reserve cannot be explained by hyperventilation, which did not take place. (From Ott, Die Sauna.)

above $39 \cdot 5^{\circ} \mathrm{C} .\left(103^{\circ} \mathrm{F}\right)$. In connexion with this and the following refrigerant, big changes of the autonomic regulations take place, as is demonstrated by analyses of blood sugar, alkali reserve, plasma cholesterol (Fig. 2) blood count, electrocardiogram (Fig 3*), etc. All these regulations are subject to the influences of the autonomic nervous system and of the endocrine glands, connected therewith. They may, therefore, be taken as indicators of the tone of the autonomic system.

In trying to understand the reactions of the body as a result of the strong thermic stimulants in and after the hot-air bath we come to the following

- See p. 200. 

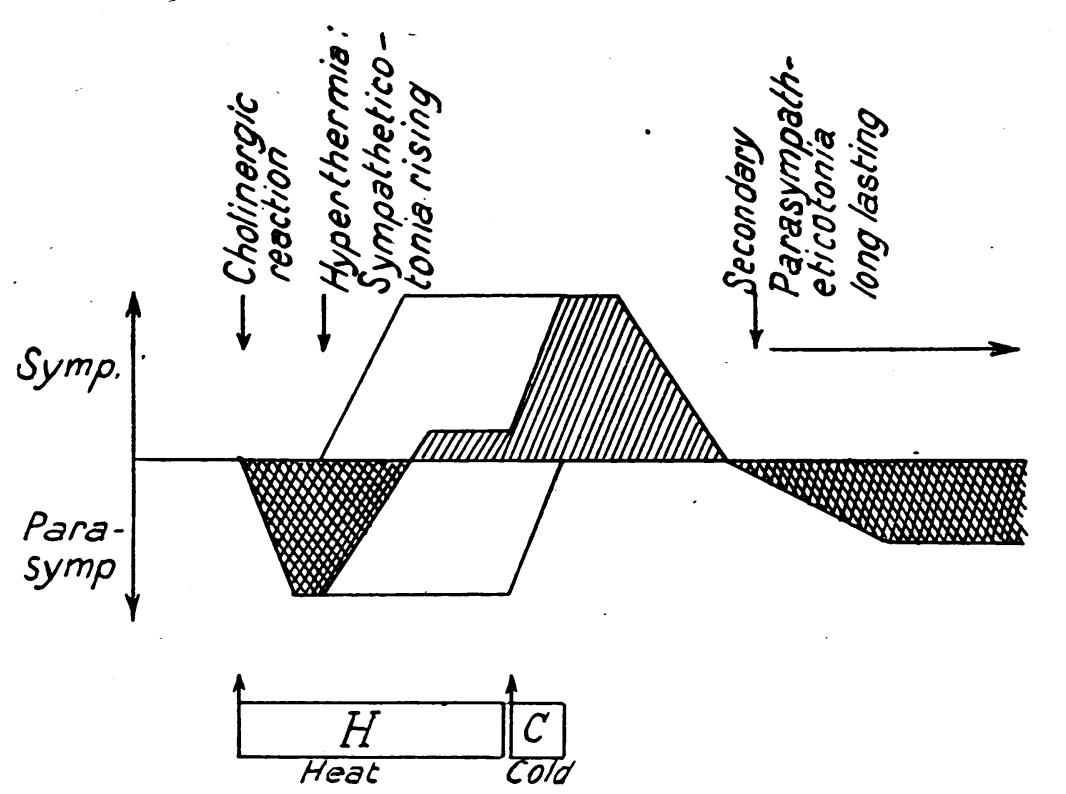

FIG. 4.-Interference of cholinergic reaction with sympatheticotonic effects produced by a combined physical treatment, such as hot-air bath (Sauna), followed by a short, cold douche. For explanations, see text. (After Ott, Die Sauna.)

conclusions (Fig. 4). Firstly, as a physiological regulation, we get a reaction with vasodilation of the skin and sweating that is to say, a cholinergic or, in the functional sense, a parasympathetic reaction. The physiological body temperature regulation is, however, for physical reasons, insufficient in the hotair room. Hyperthermia occurs, stimulating the sympathetico-adrenal system; this is particularly well demonstrated in the electrocardiogram. (As regards the sympatheticotonic variations of the electrocardiogram, we must refer to the papers of Nordenfelt, 1942; Leitner and Steinlin, 1943; Spühler, 1947: and others). At the same time the physiological regulating mechanism continues, since the thermo-regulation is still focused on the normal bases of body-temperature, a condition contrary to that found in fever with infectious diseases.

After the application of a refrigerant, the stimulation of the sympathetic system remains somewhat longer than the parasympatheticotonic tendency towards cooling; the cold stimulant may thus even cause an additional sympatheticotonia. Only later an undulating fluctuation of the autonomic tonus occurs on the other side. This parasympathetic hypertonia may last for hours or even days, as could be shown by studies on the cold reactions of the skin vessels (Jung, 1946).

These experimental findings offer a new proof that physical treatment, such as a hot-air bath, can lead to changes of the autonomic regulations which are just as strong as those that have for a long time been known to occur pathologically in the case of infectious fevers.

Secondly, they explain much better the well-known fact that reaction from physical treatment depends on the individual tonus of the autonomic system, and is much less regular than, for example, reaction to the intravenous injection of pyrogenic protein. Physical hyperthermia induces a pathological state with irritation of the sympathetico-adrenal system, the physiological cholinergic reaction being maintained at the same time (at least as long as no heat exhaustion occurs). The physical agent is, therefore, acting as an "amphotonic" stimulus, i.e. on both sides of the autonomic system, and the actual effect is dependent upon which of these two systems is reacting more easily to this stimulus. The reaction, individually as well as on the various organs, may be different. Thus it can be explained why, for example, in certain cases we may see at the same time sympatheticotonic changes of the electrocardiogram and a parasympathetic depression of the blood sugar.

Thirdly, in order to explain the empirical effect upon infectious diseases, new points of view may be shown, which may be valid not only for the hotair bath, but, in a modified form, also for other physical and hydrological measures.

It may be concluded a priori, considering the strong vegetative changes in fever, that the autonomic nervous system plays an important part in the course of natural infectious diseases. The physiologists have already for a long time been paying attention to this; I may remind readers in this respect of Cannon's (1926) emergency theory of the sympathetic system. During the last years, however, the question has to a vast extent been cleared up by experimental pathology. In this connexion, the researches of Belak (1941), W. Frei at Zurich (1945), and others have shown that a non-specific, 
quickly-working resistance reaction, such as phagocytosis and mobilization of the natural antibodies, is hastened by the sympathetic system, whereas specific immunity reactions (production of antibodies, etc.) are stimulated by the parasympathetic, that is, they are cholinergic reactions.

Now, physical therapy is a typical "reaction therapy" (von Neergaard, 1946), since it causes, as we have seen, strong reactive changes in autonomic regulation. With intensive treatments, such as the hot-air bath-i.e. with short physical hyperthermia -a short sympatheticotonia occurs, and afterwards a longer-lasting parasympatheticotonia follows. This is a special case, but with any other physical treatment, especially with spa treatment and hydrotherapy, we have to reckon with strong changes of the autonomic tonus, as has already been partly proved by experiments of various authors.

We believe that it is more important for the understanding of the effects of spa treatment and physical therapy to consider the autonomic reactions, with their effects on resistance and immunity, than to overrate the possibilities of " non-specific protein therapy", which have not yet been sufficiently investigated. These autonomic reactions also help us to inderstand why physical medicine is particularly valuable in cases of catarrhal and rheumatic diseases. We must remember that these are infections against which no specific chemotherapy or any serological treatment exist, and for which the relatively benign virus is less responsible than failure of natural resistance or immunity. The reactive changes of the tonus of the autonomic system now proves to us indirectly that by physical treatment it is possible to enhance the natural defence functions of the body against infection. Whether more stress is to be laid on a short, intensive irritation of the sympathetic system, or a longer-lasting hypertonia of the parasympathetic, is dependent on the individual disease, on the phase of the infection, and on the "starting level " of the autonomic tonus. It is thus possible to stop in its initial stage a slight infection, such as a catarrh of the respiratory tract, by activating the sympathetic, non-specific resistance reactions. Moreover, frequent stimulation of the sympathetic creates a certain training of the non-specific resistance. Subacute and chronic infections, however, may be activated by a sympatheticotonic irritation, which may prove to be useful, but in certain cases (e.g. tuberculosis) it may also be harmful. With chronic remittent infections, which interest us particularly at the moment, as, for example, chronic rheumatism, the healing is done essentially through the strengthening of specific immunity functions. In certain phases of physical therapy a distinct increase of the parasympathetic tonus may be shown, and, therefore, the enhancement of the immunity of the body by physical therapeutic measures is comprehensible from the scientific point of view also, though this had been ascertained empirically longago.

The increase of the natural resistance and immunity of the organism is, as we know, one of the principal tasks of physical medicine. The latter differs from other methods of treatment in the manner by which this aim is attained: it is not the pathogenic agent that is the object, but the reacting organism.

\section{Summary}

After a brief review on the present status of physical medicine at Swiss universities, some recent investigations at the University Institute for Phyșical Therapy in Zurich are referred to.

Rheumatism and catarrhal diseases, and the basic foundation of their physical treatment, are the principal objects of these investigations.

Statistical researches (Bruck, 1939) show the rôle of environmental influences as pathogenic factors in rheumatism, the tremendous importance of rheumatism for Swiss national economy, and the consequences of these facts in prophylaxis and social medicine.

From the clinical point of view (von Neergaard, 1939), rheumatism is not a symptomatic term but a pathological entity; its characteristic features also form a strong argument against the theory of the non-specific allergic pathogenesis of rheumatism. Focal infection is rare, and can be differentiated from "vulgar" chronic rheumatism, which has evidently an aetiology identical with the catarrhal diseases of the respiratory tract. Its pathogenesis, always depending on various factors, is a conditional one.

The function of physical methods is demonstrated by experiments made by the writer on effects of the Finnish Sauna bath. Acting as a "reaction therapy", physical methods produce changes in the tonus of the autonomic system, which may explain the therapeutic value of these treatments both in colds and allied diseases and in chronic rheumatism.

\section{REFERENCES}

Aschoff, L. (1934). Rheumaprobleme, 3, 7. Leipzig

Belak, S. (1941). Z. Immun. Forsch., 100, 264.

Bruck, M. (1939). "Bedeutung des Rheumatismus f. Volksgesundheit und Wirtschaft auf Grund schweizerischen statistischen Materials." Berne.

Cannon, W. B. (1926). Ergeb. Physiol., 27, 380.

Dietrich, S. (1938). "Rheumatische Kreislaufschädigungen." Dresden

and Leipzig. “ “Febris rheumatica." Lund.

Frei, W. (1945). Aerztl. Monatsh., 1, 70.

Frei, W. (1945). Aerztl. Monatsh., 1, 70.

Jung, W. (1946). Schweiz. med. Wschr.,

Klinge, F. (1933). Erg. Allg. Path., 27. Arch. Kreislaufforsch., 13, 62 Leergaard, K. von (1939). "Die Katarrhinfektion als chronische Allgemeinerkrankung." Dresden and Leipzig.

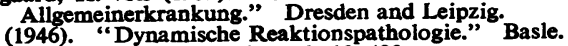

Nordenfelt, O. (1942). Nord. Med., 13, 493.

Ott; V. (1947). Die Sauna. Monograph (in preparation).

Ott; V. (1947), Die Sauna. Monograph (in preparatiz. 28.
Spühler, O. (1947). Schweiz. med. Wschr., 77, 28. 